\title{
Manipulating Liquids with Robots: a Sloshing-Free Solution
}

\author{
Lorenzo Moriello $^{\mathrm{a}, *}$, Luigi Biagiotti ${ }^{\mathrm{b}}$, Claudio Melchiorri ${ }^{\mathrm{a}}$, Andrea Paolic \\ ${ }^{a}$ Department of Electrical, Electronic and Information Engineering "Guglielmo \\ Marconi", University of Bologna, Viale del Risorgimento 2, 40136 Bologna, Italy. \\ ${ }^{b}$ Department of Engineering "Enzo Ferrari", University of Modena and Reggio Emilia, \\ via Pietro Vivarelli 10, 41125 Modena, Italy. \\ ${ }^{c}$ School of Engineering, University of Lincoln, Brayford Pool, LN6 7TS, Lincoln, UK.
}

\section{Abstract}

This paper addresses the problem of suppressing sloshing dynamics in liquid handling robotic systems by an appropriate design of position/orientation trajectories. Specifically, a dynamic system, i.e. the exponential filter, is used to filter the desired trajectory for the liquid-filled vessel moved by the robot and counteract the sloshing effect. To this aim, the vessel has been modelled as a spherical pendulum of proper mass/length subject to the accelerations imposed by the robot and the problem has been approached in terms of vibration suppression to cancel the residual oscillations of the pendulum, i.e. the pendulum swing at the end of the reference rest-to-rest motion. In addition, in order to reduce the relative motion between liquid and vessel, an orientation compensation mechanism has been devised aiming to maintain the vessel aligned with the pendulum during the motion.

\footnotetext{
*Corresponding author.

Email addresses: lorenzo.moriello2@unibo.it (Lorenzo Moriello), luigi.biagiotti@unimore.it (Luigi Biagiotti), claudio.melchiorri@unibo.it (Claudio Melchiorri), apaoli@lincoln.ac.uk (Andrea Paoli)
} 
The effectiveness of the proposed approach, both in simple point-to-point motions and complex multi-point trajectories, has been proved by means of an exhaustive set of experimental tests on an industrial manipulator that moves a cylindrical vessel filled with water.

This innovative solution effectively uses all the degrees of freedom of the robotic manipulator to successfully suppress sloshing, thus significantly improving the performances of the robotic system. Furthermore, the proposed solution, showing a high degree of robustness as well as intrinsic design simplicity, is very promising for designing novel industrial robotics applications with a short time-to-market across key manufacturing sectors (e.g., food and beverage, among others).

Keywords: Liquid Handling Robotic Systems, Sloshing Dynamics, Online Trajectory Generation, Vibration Suppression, Robotic Manipulators, Food and beverage manufacturing.

\section{Introduction}

As the current industrial scenario becomes increasingly competitive, modern manufacturing systems must comply with strict, demanding functional requirements. In this context, industrial automation plays a key role in optimizing manufacturing processes and maximizing production efficiency and flexibility, while reducing waste and energy consumption. As a consequence, industrial robotics is pervading non-traditional industrial sectors to overcome the severe and unacceptable limitations due to the use of rigid automation systems. The resulting novel use of industrial robots in non-traditional industrial sectors has created new challenges that have attracted the attention 
of scientists and technologists.

One of these key challenges is the use of robot manipulators to handle liquid materials. A particularly interesting and promising case is the use of industrial robots in the food processing industry to accomplish complex tasks - i.e., moving, manipulating, pouring liquid ingredients (see Nair (2017)) traditionally executed by human operators or by means of process control systems. In light of this, research in this area is gaining growing importance driven by the food and beverage industry needs to increase production performances, dependability and flexibility. For the same reasons, many other industrial sectors could benefit from an effective solution to this problem. As an example, the steel industry could address the problem of handling melted metal by means of industrial robots (see Aribowo et al. (2010) and Aribowo et al. (2015)).

These industrial cases can be solved by addressing the general problem of controlling a robotic manipulator to move a liquid-filled vessel without spilling. This problem is highly challenging due to the complexity of nonlinear fluid dynamics that arises within the vessel when this moves along a generic 3D trajectory. In particular, the liquid slosh dynamics - i.e., flows occurring when free surface waves are generated inside a tank - are extremely detrimental to industrial application performances and very complex to counteract.

Scientific literature has put forward a number of theoretical approaches to mathematically describe and analyse slosh dynamics (see Ibrahim (2005) and Abramson (1966) among others). Particularly, in relation to the automatic machinery sector, many solutions have been proposed to avoid sloshing 
flows when moving liquid-filled containers. Results presented in Yano and Terashima (2001), Consolini et al. (2013), Hamaguchi and Taniguchi (2002) and Hamaguchi and Taniguchi (2009) are a significant sample of existing solutions. All the methodologies used in these studies rely on the assumption of a simplified linear model of the slosh dynamics, and sloshing is tackled as a model-based disturbance suppression problem that can be solved by means of proper feed-forward or feedback control architectures. In particular, feed-forward methods, which generate a proper reference trajectory for the machine, are of industrial interest, due to their ease of implementation on standard industrial machines and their use of a reduced sensor apparatus. In addition, it is important to stress that the majority of these studies consider simple one-dimensional motions, and, even when 3D motions are introduced, the slosh phenomenon is decoupled along the different motion axes and treated as a set of independent problems (see Yano and Terashimal (2005) and Chen et al. (2007)) . Finally, when multi-degrees-of-freedom robotic manipulators are taken into consideration, the possibility of changing both the position and the orientation of the vessel - as described in Aribowo et al. (2015) and Moriello et al. (2017) - is generally ignored, despite the fact that the compensation of slosh dynamics via tilt angle modification has been proved in many papers, such as Chen et al. (2007) and Feddema et al. (1997).

This paper presents an innovative feed-forward method to effectively reduce slosh dynamics in liquid handling robotic systems. This method has been widely tested both in simulation and experimentally. The innovation of the proposed solution comes from a proper combination of two control actions: one is aimed at generating a filtered translational trajectory able 
to reduce the linear acceleration responsible for the sloshing, while the other adds to it by properly controlling the dynamical 3D orientation of the vessel. The theoretical machinery used to filter the vessel acceleration is based on the use of an exponential smoother (see Biagiotti et al. (2015)) that guarantees the required degree of continuity of the trajectory.

The effectiveness and robustness of the proposed solution proved by experimental tests, as well as its intrinsic design simplicity and ease of implementation, make this approach particularly promising for designing novel industrial robotics applications with a short time-to-market across key industrial sectors, e.g., food and beverage manufacturing.

The paper is organized as follows. In Section 2, the mathematical modelling of the slosh dynamics is briefly reviewed and an equivalent mechanical model is derived. In Section 3, the proposed solution is devised and thoroughly discussed. Finally, in Section 4 an exhaustive set of experimental results is proposed, proving the validity, effectiveness and robustness of the approach.

A preliminary version of this paper was published in the proceedings of the 2017 IEEE International Conference on Robotics and Automation (ICRA) Moriello et al. (2017).

\section{Mathematical model of the slosh dynamics}

This section aims to derive a mathematical model for the sloshing waves generated inside a liquid-filled vessel that moves along a 3D trajectory. This is a particularly complex problem that can be handled linearizing the NavierStokes equations, thus obtaining a linear model that represents the super- 
position of $j$ different sloshing modes. This topic is thoroughly discussed in Ibrahim (2005), which the present paper builds on.

This paper deals with the case of a fluid in a cylindrical vessel; however, vessels with different geometrical shapes can also be considered by simply extending the methodology.

With regard to a cylindrical vessel, the natural frequency of the $j$-th sloshing mode is

$$
\omega_{n j}=\sqrt{\frac{g \xi_{j}}{R} \tanh \left(\frac{h \xi_{j}}{R}\right)}
$$

where $g$ is the gravity constant, $R$ is the cylinder radius, $h$ is the liquid height, and $\xi_{j}$ is the $j$-th root of the derivative of the Bessel function of the first kind. Furthermore, the damping coefficient $\delta_{j}$ of the $j$-th generic sloshing mode can be derived observing how the sloshing waves lessens according to a logarithmic decay ratio due to energy dissipation. For control purposes, it is a common practice to take into account only the first asymmetric mode, which is dominant with respect to higher modes. In this case, the damping ratio is usually defined by means of an empirical relationship (see Abramson (1966)) which takes into account the kinematic viscosity $\nu$ of the fluid and the vessel parameters $h$ and $R$ :

$$
\delta_{1}=\frac{2.89}{\pi} \sqrt{\frac{\nu}{\sqrt{R^{3} g}}}\left[1+\frac{0.318}{\sinh \left(\frac{1.84 h}{R}\right)} \frac{1-\frac{h}{R}}{\cosh \left(\frac{1.84 h}{R}\right)}\right] .
$$

This linear model defines a mechanical system that approximates the liquid dynamics in a moving vessel. The equivalent model consists of a rigid mass $m_{0}$ and a series of pendula with mass $m_{j}$, length $l_{j}$, and support point 


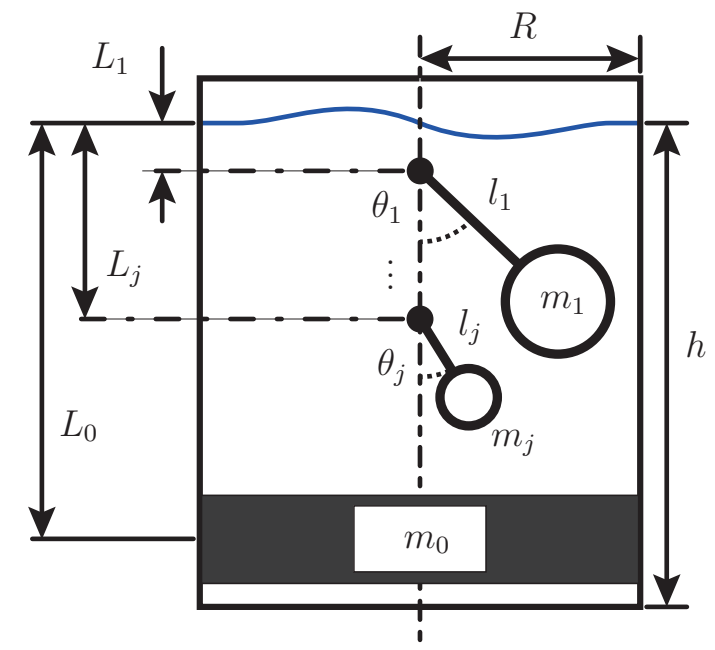

Figure 1: Equivalent mechanical model approximating the liquid dynamics in a cylindrical vessel.

located at a distance $L_{j}$ from the undisturbed free surface of the liquid. Figure 1 depicts this mechanical system.

Since the equivalent mechanical model must preserve the mass and the momentum of inertia of the liquid, as well as possess the same modes of oscillations as the original system, a set of relations between the physical parameters $m_{j}, l_{j}, L_{j}$ and the modes characteristics $\omega_{n j}$ and $\delta_{n j}$ can be derived (see Ibrahim (2005)). As an example, the pendulum relation $\omega_{n j}=$ $\sqrt{g / l_{j}}$ allows to derive the length $l_{j}$. These relations, here omitted for the sake of brevity and clarity, show that the values of $L_{j}$ are rather small, and consequently the pivots of the pendula lie near the liquid surface. In addition, both the lengths $l_{j}$ and masses $m_{j}$ rapidly decrease as the index $j$ grows. In view of this result, this paper proposes a simplified model of the slosh dynamics. Specifically, this simplified model only describes the first asymmetric mode of the slosh by means of a single pendulum with mass $m_{1}$, 


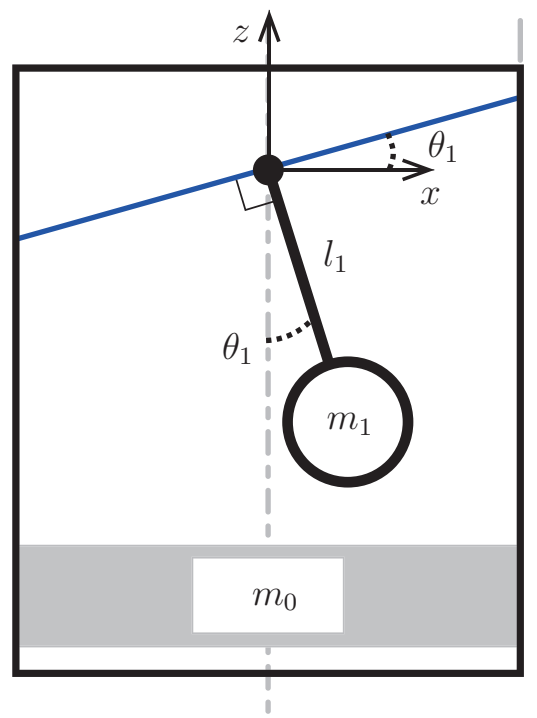

Figure 2: Simplified mechanical model approximating the first asymmetric sloshing mode in a cylindrical vessel.

length $l_{1}$ and pivot located at the centre of the liquid surface. Moreover, the pendulum is assumed to be always orthogonal to the liquid surface that is supposed to be planar. Based on this assumption, the pendulum angle $\theta_{1}$ measures the angle between the liquid surface and the horizon. Figure 2 illustrates this simplified mechanical model.

When the liquid-filled vessel is moved by a robotic manipulator, the effect of the 3D motion on the pendulum pivot needs to be modelled. To this aim, a spherical pendulum is assumed to model the sloshing dynamics in the vessel. In this framework, the configuration of the pendulum in the space can be described by the generalized coordinates vector $[x, y, z, \varphi, \theta]^{T}$, where $\varphi$ and $\theta$ are the angles that define a spherical coordinate system and $[x, y, z]^{T}$ is the position of the pendulum pivot. 


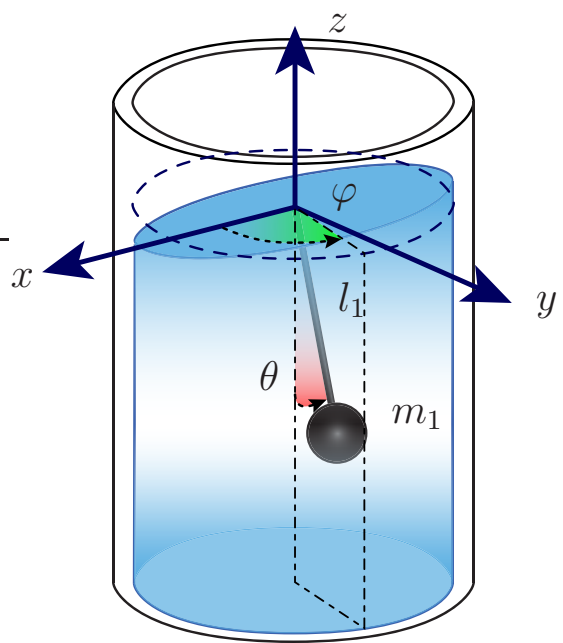

Figure 3: Spherical pendulum modelling the sloshing dynamics in a liquid-filled vessel that moves along a $3 \mathrm{D}$ trajectory.

In this scenario, shown in Figure 3, the position of the mass $m_{1}$ is

$$
\left[\begin{array}{l}
x_{m_{1}} \\
y_{m_{1}} \\
z_{m_{1}}
\end{array}\right]=\left[\begin{array}{l}
x \\
y \\
z
\end{array}\right]+\operatorname{Rot}_{z}(\varphi) \operatorname{Rot}_{y}(-\theta)\left[\begin{array}{c}
0 \\
0 \\
-l_{1}
\end{array}\right]=\left[\begin{array}{c}
x+l_{1} \cos (\varphi) \sin (\theta) \\
y+l_{1} \sin (\varphi) \sin (\theta) \\
z-l_{1} \cos (\theta)
\end{array}\right]
$$

As a result, the kinetic energy of the mass $m_{1}$ is given by

$$
\begin{aligned}
T= & \frac{1}{2} m_{1}\left[\dot{x}_{m_{1}}^{2}+\dot{y}_{m_{1}}^{2}+\dot{z}_{m_{1}}^{2}\right] \\
= & \frac{1}{2} m_{1}\left[\left(\dot{x}-l_{1} \sin (\varphi) \sin (\theta) \dot{\varphi}+l_{1} \cos (\varphi) \cos (\theta) \dot{\theta}\right)^{2}\right. \\
& +\left(\dot{x}-l_{1} \sin (\varphi) \sin (\theta) \dot{\varphi}+l_{1} \cos (\varphi) \cos (\theta) \dot{\theta}\right)^{2} \\
& \left.+\left(\dot{z}+l_{1} \sin (\theta) \dot{\theta}\right)^{2}\right]
\end{aligned}
$$


while its potential energy is equal to

$$
V=m_{1} g z_{m_{1}}=m_{1} g\left(z-l_{1} \cos (\theta)\right)
$$

where $g$ is the gravity acceleration.

Considering the Lagrangian function $\mathcal{L}=T-V$ and Lagrange's equations for the generalized coordinates $\varphi$ and $\theta$

$$
\begin{aligned}
& \frac{d}{d t}\left(\frac{\partial \mathcal{L}}{\partial \dot{\varphi}}\right)-\frac{\partial \mathcal{L}}{\partial \varphi}=0 \\
& \frac{d}{d t}\left(\frac{\partial \mathcal{L}}{\partial \dot{\theta}}\right)-\frac{\partial \mathcal{L}}{\partial \theta}=0
\end{aligned}
$$

it is possible to derive the following motion equations for the spherical pendulum:

$$
\begin{array}{rr}
l_{1} m_{1} \sin (\varphi)\left(l_{1} \sin (\theta) \ddot{\varphi}+2 l_{1} \cos (\theta) \dot{\varphi} \dot{\theta}-\sin (\varphi) \ddot{x}+\cos (\varphi) \ddot{y}\right)=0 \\
l_{1} m_{1}\left(l_{1} \ddot{\theta}-l_{1} \cos (\theta) \sin (\theta) \dot{\varphi}^{2}+\cos (\theta)(\cos (\varphi) \ddot{x}+\sin (\varphi) \ddot{y})+\sin (\theta)(g+\ddot{z})\right) \\
=0 .
\end{array}
$$

Particularly important is equation (5) that represents the swing of the pendulum and, accordingly, the sloshing dynamics. 


\section{A feedforward approach to suppress sloshing}

This section presents a control strategy that allows to suppress sloshing in a liquid-filled vessel moved by a robot manipulator. This problem could be particularly challenging using a standard feedback control architecture, as the controller should instantly react to the high speed dynamics of the fluid. This control strategy would require extremely fast and accurate sensors, as well as complex, non-linear, and usually non-robust algorithms. Differently from this approach, this paper puts forward a simple and robust feedforward controller that can be easily and successfully implemented in industrial applications. To this aim, a two-stage controller is derived starting from the spherical pendulum dynamics (5) that model the sloshing effect. Specifically, the former stage counteracts the oscillations of the pendulum by smoothing the trajectory by means of an exponential filter, while the latter is an orientation compensation mechanism aimed at maintaining the vessel aligned with the virtual pendulum during the motion.

\subsection{Sloshing compensation via trajectory smoothing}

As anticipated in the previous section, sloshing dynamics can be suppressed by solving an oscillation suppression problem on the mechanical model of the virtual pendulum. To do this, the pendulum swing dynamics (5) is linearized for small angular variations around the equilibrium point

$\dot{\theta}=\theta=\dot{\varphi}=0, \varphi=\bar{\varphi}$, where $\bar{\varphi}$ is a generic constant angular value. The obtained linear model is given by

$$
\ddot{\theta}+\frac{c}{m_{1} l_{1}^{2}} \dot{\theta}+\frac{g}{l_{1}} \theta=-\frac{1}{l_{1}}(\cos (\bar{\varphi}) \ddot{x}+\sin (\bar{\varphi}) \ddot{y})
$$


where an additional dissipation term has been introduced. This model is equivalent to the general second order equation

$$
\ddot{\theta}+2 \delta_{1} \omega_{n, 1} \dot{\theta}+\omega_{n_{1}}^{2} \theta=u
$$

where $\omega_{n, 1}$ and $\delta_{1}$ are defined by equations (11) and (2) respectively, and the input $u$ is a linear combination of the accelerations along the $x$ and $y$ axes.

In light of this result, the proposed solution builds on Biagiotti et al. (2015), where the authors present a simple and effective method to reduce the oscillations of the vibratory servo system

$$
G(s)=\frac{\omega_{n}^{2}}{s^{2}+2 \delta \omega_{n} s+\omega_{n}^{2}}
$$

by applying a proper feed-forward control on the reference signal. Specifically, Biagiotti et al. (2015) proves that by placing an exponential filter 1

$$
F_{e x p}(s)=\frac{\sigma}{e^{\sigma T}-1} \frac{1-e^{\sigma T} e^{-T s}}{s-\sigma}
$$

in series with the system $G(s)$, it is possible to perfectly suppress the residual vibration if the following conditions hold:

$$
\begin{aligned}
\sigma & =-\delta \omega_{n} \\
T & =\frac{2 \pi}{\omega_{n} \sqrt{1-\delta^{2}}} .
\end{aligned}
$$

\footnotetext{
${ }^{1}$ Note that $F_{\text {exp }}(s)$ is described as "exponential filter" because its impulse response, which can be derived by anti-transforming (8), is a truncated exponential function with decay rate $\sigma$ and duration $T$.
} 
This result can be demonstrated by analysing the poles and zeros of the transfer function $F_{\text {exp }}(s)$ when the conditions (9) and (10) are both met. In this case, the exponential filter has a real pole in $p=-\delta \omega_{n}$, while the zeros - derived solving the equation $1-e^{\sigma T} e^{-T s}=0-$ are $z_{n}=-\delta \omega_{n} \pm$ $j n \omega_{n} \sqrt{1-\delta^{2}}, n=0,1, \ldots$ (see Figure 4). This property shows that the filter $F_{e x p}(s)$ introduces an infinite number of zeros located along a vertical line in the complex plane. In particular, the zeros obtained when $n=1$ cancel the poles of the plant $G(s)$, which allows a perfect compensation of the vibrating dynamics. It is important to stress that this cancellation, and consequently the regulation effect of $F_{\exp }(s)$, is independent of additional (possibly non-modelled) modes or zeros of the plant. In addition. Biagiotti et al. (2015) shows that, even if a complete vibration suppression is possible only when system parameters are perfectly known, the residual vibration is significantly reduced in case of parameters mismatch. Finally, it is important to point out that the effectiveness of the exponential filter does not depend on the particular input used. As a matter of fact, the filtered trajectory preserves the desired steady states and is characterized by increased smoothness. The former property is due to the unitary static gain of the exponential filter, while the latter is due to the fact that the filter increases the number of continuous derivatives of the trajectory.

Considering this result, the exponential filters is used to smooth the robot trajectory and counteract the sloshing effect within the vessel. Specifically, since the input signal $u$ in the simplified mechanical model (6) is a linear combination of the accelerations $\ddot{x}$ and $\ddot{y}$ applied to the vessel by the robot, the desired filtering action can be obtained by independently filtering the 


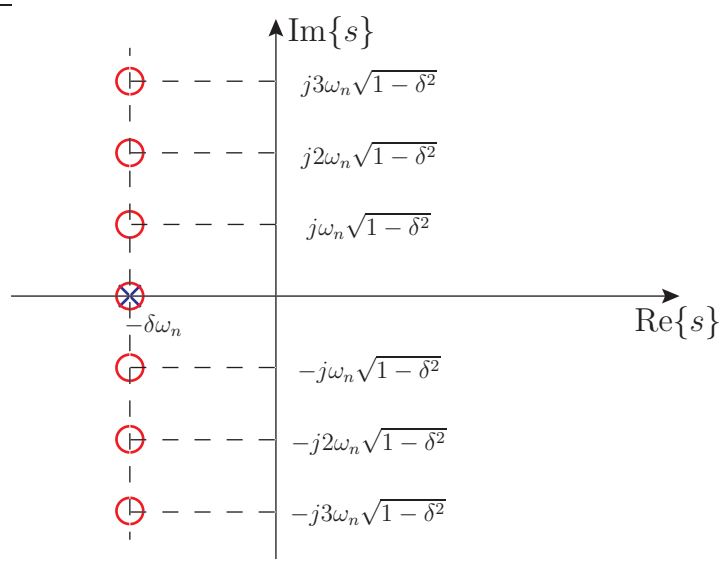

Figure 4: Pole-zero map of $F_{\text {exp }}(s)$.

robot trajectory components along the $x$ and $y$ directions. In addition, it is important to highlight that one side effect of the exponential filter is to introduce a $T$ delay into the filtered signal. For this reason, to avoid any distortion, the exponential filter is applied to the complete robot trajectory, including the component along the $z$ direction. To conclude, the exponential filter is designed to compensate only the dominant sloshing mode at frequency $\omega_{n 1}$. However, because of the low-pass characteristics of its sensitivity curve with respect to $\omega_{n}$, the exponential filter can also compensate secondary sloshing modes at higher frequencies. This point is discussed in more depth in Section 4.3, where the behaviour of the exponential smoothers is compared with that of different filtering methods, i.e. the ZVD input shapers.

The efficacy of the exponential filter is shown in Figure 5 which depicts the simulated dynamical behaviour of a cylindrical vessel. It has a radius $R=97.5 \mathrm{~mm}$, it is filled with 3 litres of water 2 , and it is subject to a $1 \mathrm{~m}$

\footnotetext{
${ }^{2}$ Kinematic viscosity of water is $\nu_{w}=1.004^{-6} \mathrm{~m}^{2} / \mathrm{s}$.
} 
translation along the $y$ direction. Importantly, Figure 5 shows a comparison between the oscillatory dynamics of the mechanical system when a double-S velocity trajectory is applied (black solid line) and when the same input is filtered (blue dashed line). In both cases, the reference trajectory is compliant with the following kinematic constraints on the derivatives: $\left|V_{\max }\right|=1 \mathrm{~m} / \mathrm{s}$; $\left|A_{\max }\right|=2 \mathrm{~m} / \mathrm{s}^{2}$; and $\left|J_{\max }\right|=10 \mathrm{~m} / \mathrm{s}^{3}$. The simulations are performed using MATLAB Simscape with mechanical parameters derived according to the method presented in Section 2 .

While the spherical coordinate system based on angles $(\theta, \varphi)$ is very effective to describe the configuration of the pendulum modelling sloshing in a 3D space, it may be misleading to understand simulative and experimental results. As an example, Figure 3 shows that the angle $\theta$, representing the swinging motion of the pendulum, ranges from 0 to $\pi$ (and not from $-\pi$ and $\pi$ ) even if the pendulum performs a planar motion. As a matter of fact, a thorough analysis of the sloshing dynamics in a 3D space cannot leave aside the angle $\varphi$. However, since the aim of this work is to reduce sloshing amplitude regardless of sloshing phase, only angle $\theta$ is taken into consideration.

Simulations of Figure 5 show that the exponential filter effectively reduces the slosh dynamics when the vessel stops in the final position. Any small residual oscillations are due to the neglected nonlinear dynamics of the mechanical pendulum. However, when the vessel moves, the liquid surface is subject to large oscillations which are due to the acceleration along the $y$ direction. In order to compensate this effect, the next subsection presents a method that uses robot degrees of freedom to control the vessel orientation. 


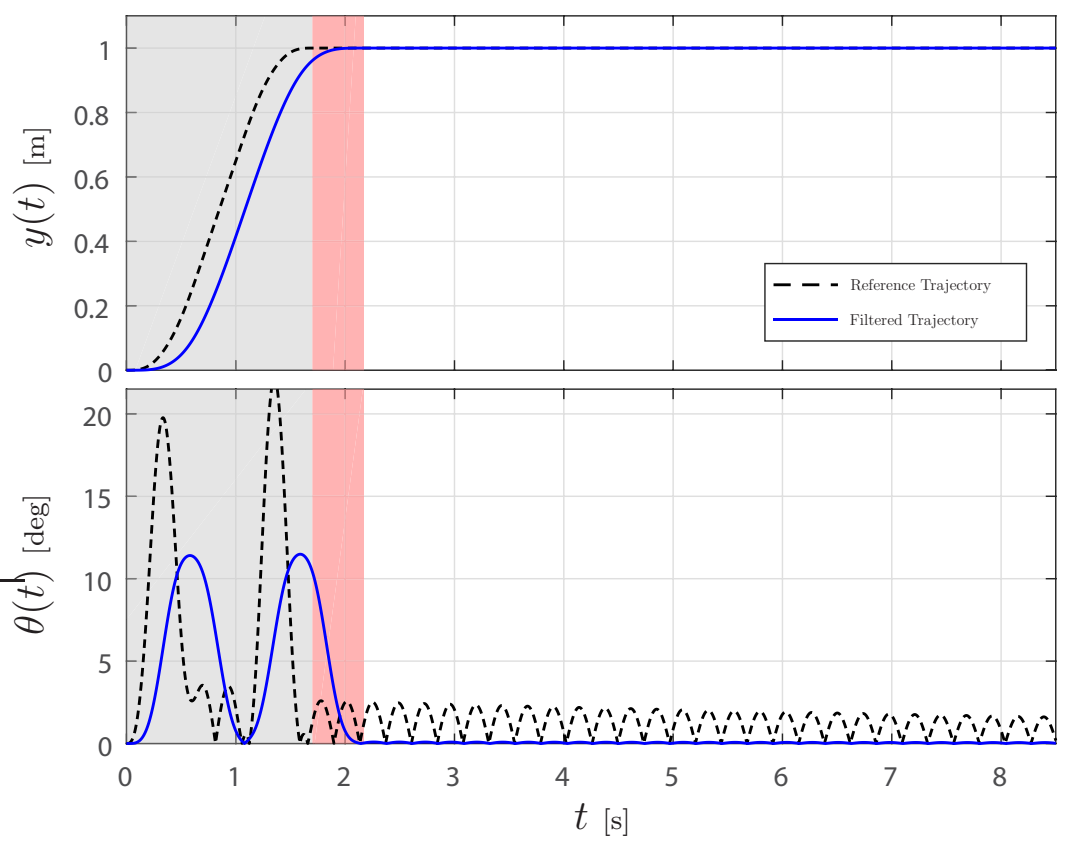

Figure 5: Simulated dynamical behaviour of a cylindrical vessel, with radius $R=97.5$ $\mathrm{mm}$ and filled with 3 litres of water, subject to a $1 \mathrm{~m}$ translation along the $y$ direction. The velocity follows a double-S trajectory with the following kinematic constraints on the derivatives: $\left|V_{\max }\right|=1 \mathrm{~m} / \mathrm{s} ;\left|A_{\max }\right|=2 \mathrm{~m} / \mathrm{s}^{2} ;$ and $\left|J_{\max }\right|=10 \mathrm{~m} / \mathrm{s}^{3}$. The black dashed lines illustrate the dynamics when no filter is utilized, while the blue solid lines show the dynamics when the input is smoothed via the exponential filter. The grey area indicates the duration of the reference trajectory, while the red area highlights the delay caused by the exponential filter.

\subsection{Total sloshing elimination using tilting compensation}

As shown in Figure 5, total elimination of the sloshing phenomenon can only occur if the relative motion between the liquid and the vessel is compensated. This result, as illustrated in Figure 6, can be achieved by designing a tilting compensation mechanism that instantaneously controls the angles $\theta$ and $\varphi$ to align the vessel with the virtual pendulum during the motion. This control action is derived by adapting the approach proposed in Chen et al. (2007) and Feddema et al. (1997). 


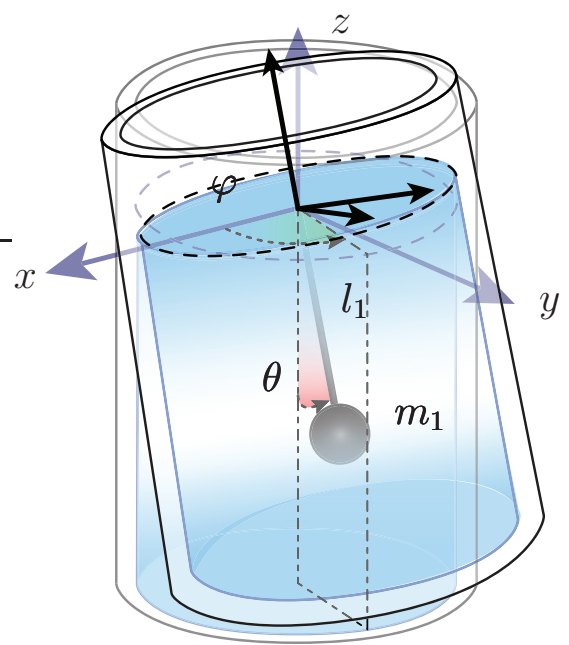

Figure 6: Aligning the vessel with the virtual pendulum using tilting compensation.

Starting from the spherical pendulum dynamics, the value of the sloshing angles is estimated from the input-output relation derived from the nonlinear differential equations (4) and (5):

$$
\begin{aligned}
& -\sin (\varphi) \ddot{x}+\cos (\varphi) \ddot{y}=0 \\
& \cos (\theta)(\cos (\varphi) \ddot{x}+\sin (\varphi) \ddot{y})+\sin (\theta)(g+\ddot{z})=0 .
\end{aligned}
$$

Solving equations (11) and (12), the necessary angular values can be determined as

$$
\begin{aligned}
& \theta=\tan ^{-1}\left(\frac{\sqrt{\ddot{x}^{2}+\ddot{y}^{2}}}{g+\ddot{z}}\right) \\
& \varphi=\pi+\operatorname{atan} 2(\ddot{y}, \ddot{x})
\end{aligned}
$$

where atan2 is the four quadrant inverse tangent. Note that equations (13) 
and (14) guarantee that $0 \leq \theta \leq \pi$ and $0 \leq \varphi \leq 2 \pi$.

As a consequence, controlling the vessel orientation by the two consecutive rotations $\operatorname{Rot}_{z}(\varphi)$ and $\operatorname{Rot}_{y}(-\theta)$ allows to align the vessel with the virtual pendulum. However, the first rotation about the $z$ axis causes an undesired vessel rotation of an angle $\varphi$ about its symmetry axis. This effect can be compensated by introducing an additional rotation about the $z$ axis. Therefore, the desired final configuration of the vessel is defined as

$$
\mathbf{R}(\theta, \varphi)=\operatorname{Rot}_{z}(\varphi) \operatorname{Rot}_{y}(-\theta) \operatorname{Rot}_{z}(-\varphi)
$$

It is worth emphasizing that the term $\operatorname{Rot}_{z}(-\varphi)$ offers an additional advantage when $\ddot{y}=\ddot{x}=0$. In this case, the angle $\varphi$ in (14) is not well defined; however, since $\theta=0$, then $\mathbf{R}(\theta, \varphi)=\operatorname{Rot}_{z}(\varphi) \operatorname{Rot}_{z}(-\varphi)=I_{3}$, where $I_{3}$ is a $3-$ by -3 identity matrix.

Importantly, since the angles $\theta$ and $\varphi$ depend on the translational accelerations $\ddot{x}$ and $\ddot{y}$, the continuity of the orientation trajectory depends on the continuity level of the acceleration profiles. In this respect, the proposed methodology benefits from the use of the exponential filter $F_{\exp }(s)$ that increases the continuity level of the filtered signal. As a matter of fact, when the exponential filter is applied to a double-S velocity trajectory or a cubic spline trajectory 3 , it generates functions of class $\mathbb{C}^{3}$. For this reason, the acceleration profiles $\ddot{x}$ and $\ddot{y}$, as well as the orientation profiles defined according to equations (13) and (14), are class $\mathbb{C}^{1}$ functions. This property

\footnotetext{
${ }^{3}$ Double-S and cubic spline trajectories are standard robot trajectories for point-topoint and multi-point motions.
} 

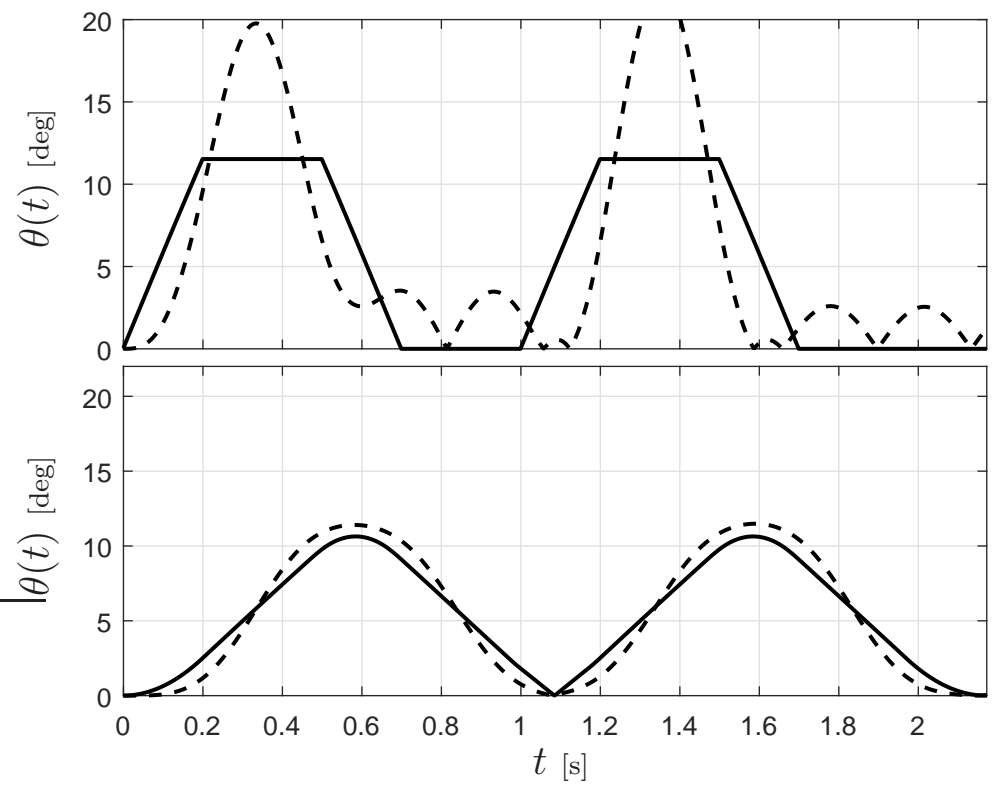

Figure 7: Simulative comparison between the angle $\theta$ derived from equation (13) (solid line) and the output of the simulated spherical pendulum (dashed line). The top plot illustrates the case of non-filtered trajectories, while the bottom plot depicts the case of filtered trajectories.

guarantees that the angular trajectories are feasible for a robot manipulator to track, which differs from Chen et al. (2007), where an additional average filter must be applied to the reference input with the only purpose of smoothing the acceleration profiles.

To prove the efficacy of the tilting compensation mechanism, Figure 7 shows a simulative comparison between the angle $\theta$ derived from equation (13) (solid line) and the output of the simulated spherical pendulum (dashed line). In both cases, the same acceleration profiles as in Figure 5 is considered. The top plot illustrates the case of non-filtered trajectories, while the bottom plot depicts the case of filtered trajectories. 


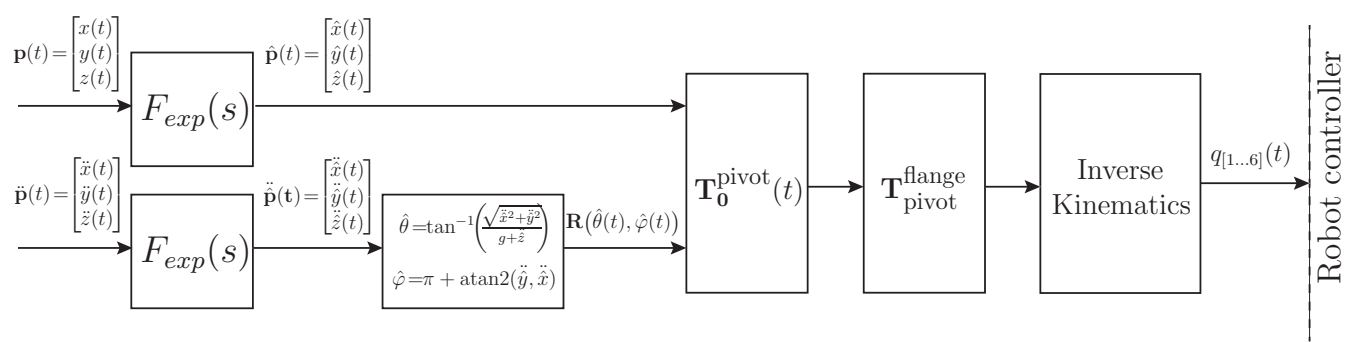

Figure 8: Feedforward controller for sloshing compensation.

\subsection{Feedforward controller for sloshing compensation}

As shown in Figure 8, the overall feedforward control architecture is obtained by combining the smoothing action and the tilting compensation mechanism. Specifically, the position and orientation profile of the centre of the liquid surface, corresponding to the trajectory of the pivot of the virtual pendulum, are derived from the desired trajectory profile of the liquid container $\mathbf{p}(t), \dot{\mathbf{p}}(t)$ and $\ddot{\mathbf{p}}(t)$. The pivot trajectory is then filtered by the exponential filter $F_{\text {exp }}(s)$ to obtain the filtered pivot trajectory $\hat{\mathbf{p}}(t), \dot{\hat{\mathbf{p}}}(\mathbf{t})$ and $\ddot{\hat{\mathbf{p}}}(\mathbf{t})$. Furthermore, the values of the desired vessel orientation angles $\hat{\theta}(t)$ and $\hat{\varphi}(t)$ are derived from $\ddot{\hat{\mathbf{p}}}(\mathbf{t})$ by means of equations (13) and (14), and are used to define the rotation matrix $\mathbf{R}(\hat{\theta}(t), \hat{\varphi}(t))$. Therefore, trajectories $\hat{\mathbf{p}}(t)$ and $\mathbf{R}(\hat{\theta}(t), \hat{\varphi}(t))$ can be organized in the homogeneous transformation matrix

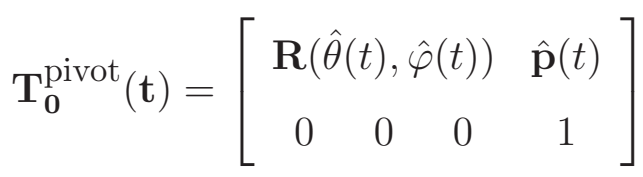

that instantly provides the desired configuration of a reference frame attached to the liquid surface with respect to the world reference frame. Finally, the additional homogeneous transformation matrix $\mathbf{T}_{\text {pivot }}^{\text {flange }}$ is used to take into account the relative pose of the robot flange with respect to the liquid sur- 
face. The obtained trajectory, representing the set of configurations that the robot must track, is then processed using the inverse kinematics to derive the joint trajectories $q_{[1 \ldots 6]}(t)$ that feed the robot controller.

It is important to note that, when the sloshing mode is poorly damped, as is the case of the experimental tests presented in this paper, the exponential filter $F_{\exp }(s)$ becomes a rectangular smoother, i.e. a standard element to obtain multi-segment polynomial trajectories, such as double-S velocity trajectories (see Biagiotti et al. (2015) for additional details). In light of this result, sloshing compensation could also be obtained by a proper design of the vessel motion trajectories, as proposed in Biagiotti and Melchiorri (2012) and, later on, in Besset and Béarée (2017). On the one hand this property would simplify the controller structure, on the other hand it would limit the motion possibilities. For this reason, the controller structure based on the exponential smoother, which can be applied to any trajectory, is less conservative and therefore preferable. Obviously, in point-to-point motions the use of properly defined multi-segment polynomial trajectories can further enhance the capability of the system to suppress the sloshing.

\section{Experimental results}

This section presents the experiments conducted to validate the proposed feedforward architecture.

\subsection{Experimental setup}

The proposed solution has been tested using the experimental setup shown in Figure 9. The setup is based on the COMAU Smart5 Six industrial robotic arm controlled by the COMAU C4G Controller and interfaced with a 
standard PC equipped with an Intel Core 2 Duo $2.4 \mathrm{GHz}$ processor and $1 \mathrm{~GB}$ of RAM. The COMAU Smart5 Six anthropomorphic robot has 6 degrees of freedom and $6 \mathrm{Kg}$ payload. The COMAU C4G Controller performs both the position/velocity adaptive control and the power stage management, which implements the current control loop of each joint. In addition, the C4G Controller allows the integration of the robot control unit with an external PC through the software functionality "C4G OPEN". This feature has been used to implement the proposed control structure on top of the robot standard control algorithms. The C4G OPEN architecture guarantees real time Ethernet communication with the PC that runs the RTAI-Linux 3.9 operating system on a Ubuntu NATTY distribution with Linux kernel 2.6.38.8. The feedforwad controller shown in Figure 8 has been implemented using Matlab/Simulink with a sampling period equal to $T_{s}=2 \mathrm{~ms}$. The exponential filter is discretized with the sampling time $T_{s}$ by means of a discrete FIR filter $F_{\text {exp }}(z)$ derived by Z-transforming the transfer function $F_{\exp }(s)$ in equation 8 (see Biagiotti et al. (2015) for additional details).

To complete the setup, a cylindrical steel pot of radius $97.5 \mathrm{~mm}$ has been rigidly attached to the robot flange. In addition, a stiff structure has been designed and built to support an ASUS Xtion PRO Live RGB-D camera used to monitor the liquid surface during the tests. The container has been filled with water with the addition of acrylic blue dye, in order to facilitate the monitoring of the liquid surface through the camera. The RGB-D camera is connected to a laptop running ROS, which is used for video acquisition and off-line image processing. This vision system is able to acquire both RGB and depth video streams at a frame rate of $30 \mathrm{fps}$. Using Matlab, the depth 


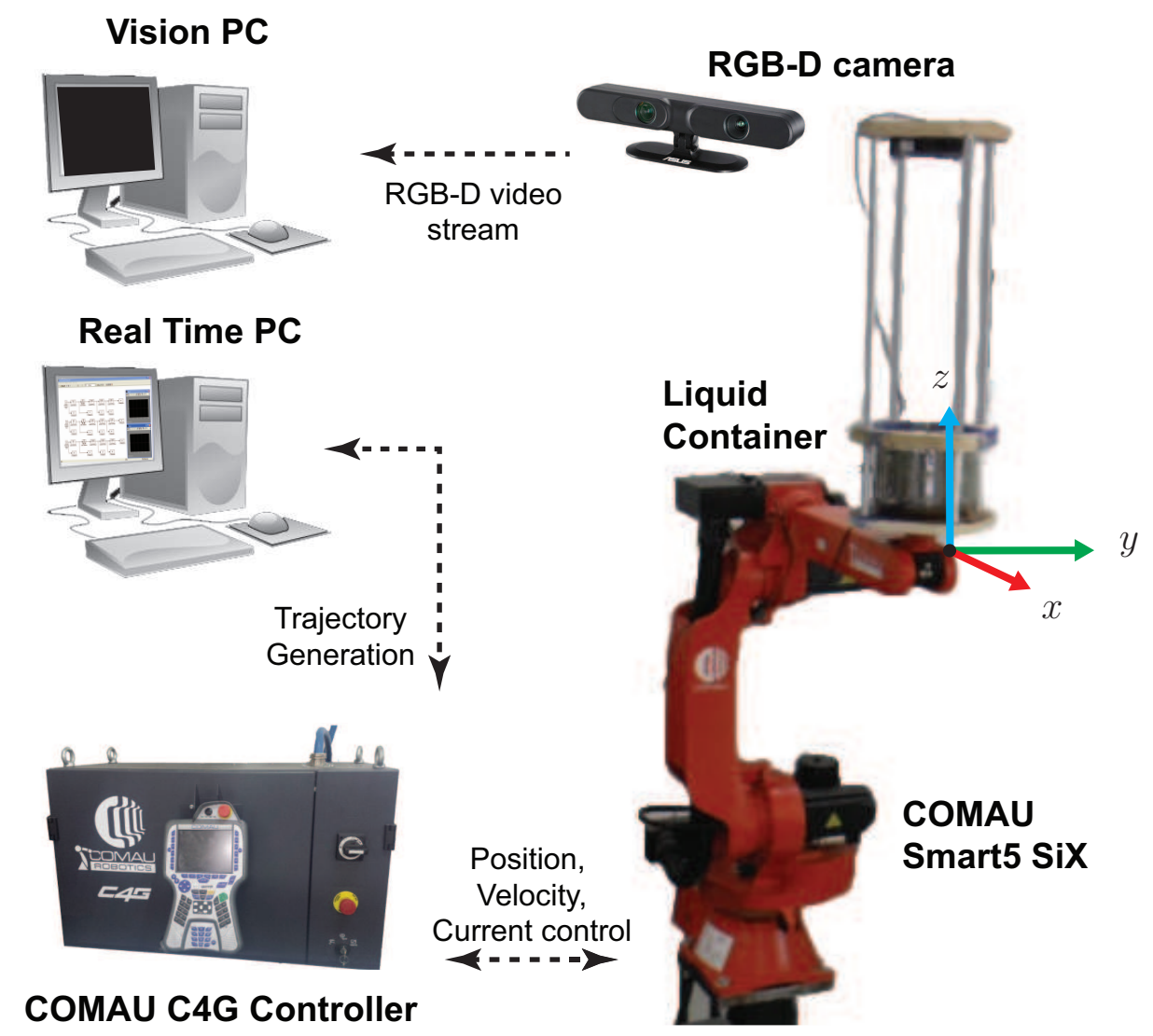

Figure 9: Experimental setup used to test the proposed solution.

video stream acquired by the RGB-D camera has been analysed to derive information about the motion of the liquid surface. Specifically, since the liquid free-surface is assumed to be a plane, each raw depth frame has been processed in order to find the interpolating plane. This is obtained using the Matlab function fit to derive the parameters $(\Delta h, K x, K y)$ that define the plane

$$
z=\Delta h+K_{x} x+K_{y} y
$$

that best fits the depth map of each frame. In this way, it is possible to 


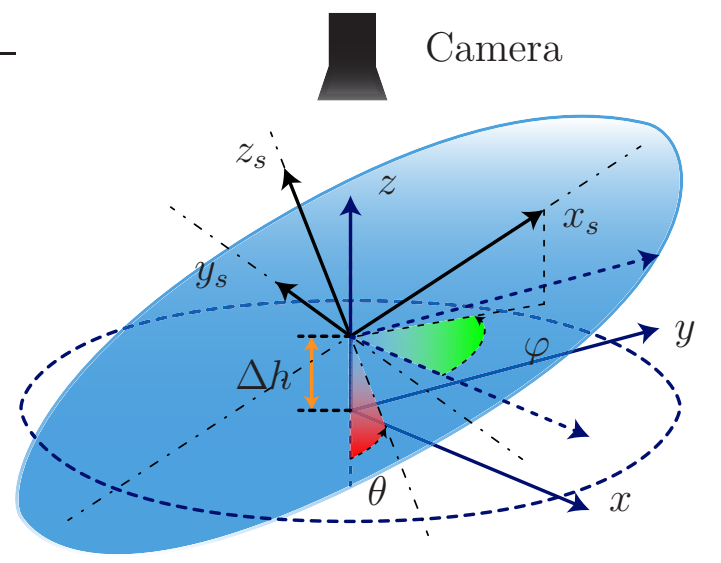

Figure 10: Liquid surface reconstruction by means of a $2 \mathrm{D}$ interpolation of the depth measurements coming from the RGB-D camera.

analyse the instantaneous motion of the liquid surface when the robot moves. Finally, the liquid surface orientation has been transformed in terms of spherical coordinates

$$
\varphi=\operatorname{atan} 2\left(K_{y}, K_{x}\right), \quad \theta=\arctan \left(K_{x} \cos \varphi+K_{y} \sin \varphi\right)
$$

Figure 10 shows the parameters used to derive the liquid surface orientation. It is important to emphasize that the RGB-D camera is only used for inspection and measurement purposes, without any intent of introducing a feedback control action.

\subsection{Experimental validation of the approximated sloshing model}

A first set of tests has been performed to derive the parameters of the pendulum model representing the first sloshing mode, as described by equation ([6). To this aim, the robot, carrying different quantities of water, has been commanded to follow a simple third order point-to-point motion along 
the $y$ axis of the world reference frame. It is important to note that this set of tests experimentally validate the theoretical model described in Section 2 . Specifically, the double-S velocity profile used in the experiments is depicted with a black dashed line in Figure 11.

This trajectory has been filtered using both the exponential smoother $F_{\text {exp }}(z)$ and the ZVD input shaper $F_{z v d}(z)$ (see the blue solid line and the dashdot red line in Figure 11). ZVD input shapers are the standard trajectory filters used in literature for residual vibrations suppression (see Singer et al. (1999)), which explains why they have been chosen in these tests. In addition, as shown in Figure 11, the ZVD shaper introduces the same delay $T$ as the exponential filter. Figure 12 illustrates the response of the vessel filled with 3 litres of water to the non-filtered signal. For the sake of clarity, only the inclination angle $\theta$ is shown, $\theta$ being defined as the polar angle along the direction of maximum inclination (i.e. the azimuth angle $\varphi$ ), which makes it the best metrics for measuring the sloshing phenomenon. It is important to note that the variable $\theta$ is always positive here, unlike the case of the classical representation of a planar pendulum angle. Furthermore, $\theta$ is characterized by an oscillation period which is half of the standard period of a pendulum swing. In light of these results, the parameters $\omega_{n 1}[\mathrm{rad} / \mathrm{s}]$ and $\delta_{1}$ of the linear system (6) can be easily derived applying a simple identification technique to the system response (see Biagiotti et al. (2015)).

Table 1 shows a comparison between the parameters experimentally obtained for different liquid volumes and their theoretical values derived from equations (11) and (2). It is important to highlight that the difference between the experimental and numerical values of the natural frequency $\omega_{n 1}$ is lower 

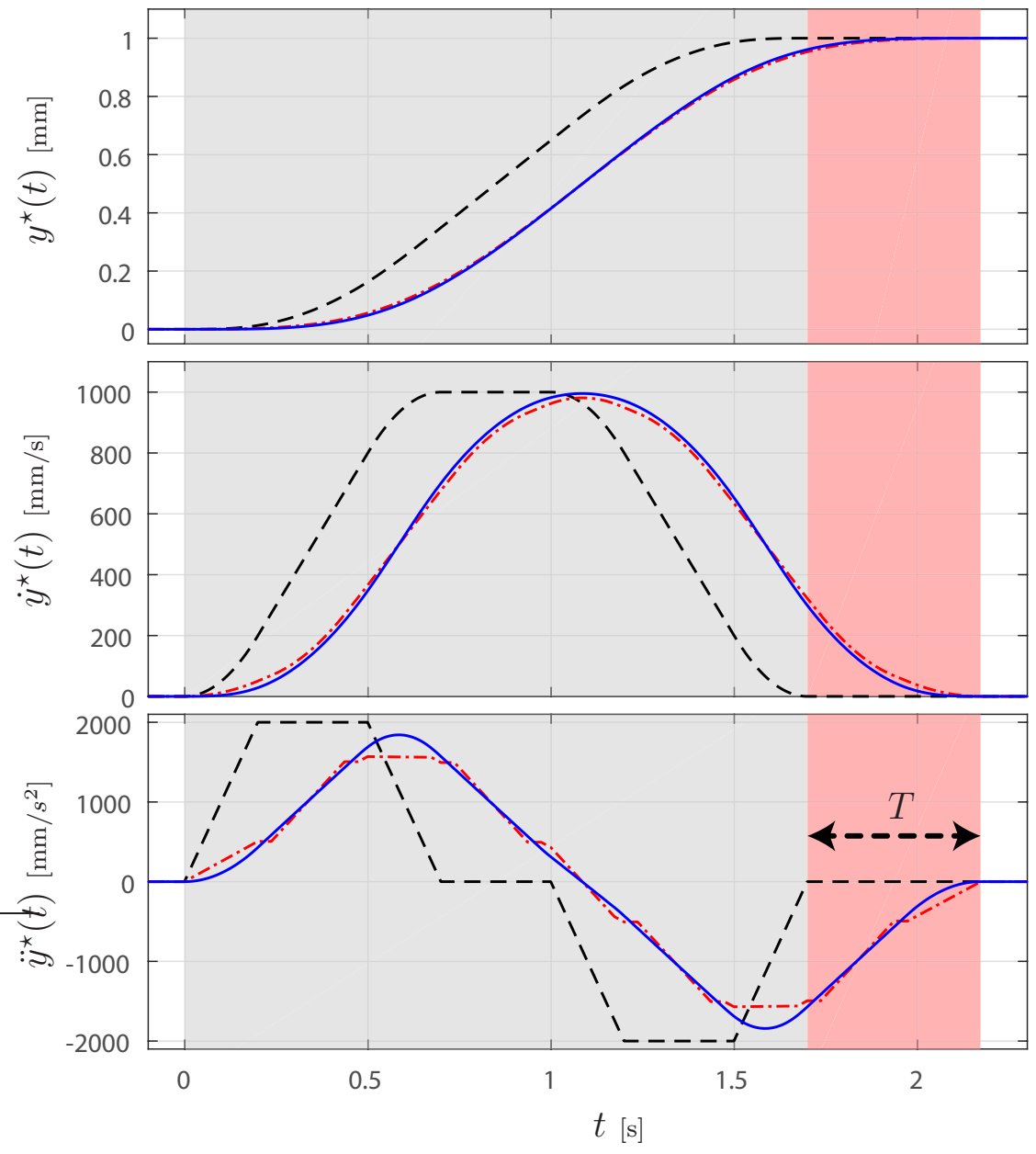

Figure 11: Trajectory used to validate the approximated sloshing model. The third order trajectory set-point is depicted with a black dashed line. This trajectory filtered with the exponential smoother $F_{\exp }(z)$ and the same trajectory filtered with a ZVD input shaper $F_{z v d}(z)$ are respectively illustrated with a blue solid line and a dashdot red line. The red and grey zones indicate the time duration of the trajectories with and without the filtering actions.

than $2 \%$. Instead, taking into account the damping ratio $\delta_{1}$, the same difference turns out to be approximately $40 \%$. It is necessary to emphasize that, since the filtering efficacy of $F_{\text {exp }}(z)$ strongly depends on $\omega_{n}$ and is much less sensitive to $\delta$, the proposed theoretical model can be used with reasonable 


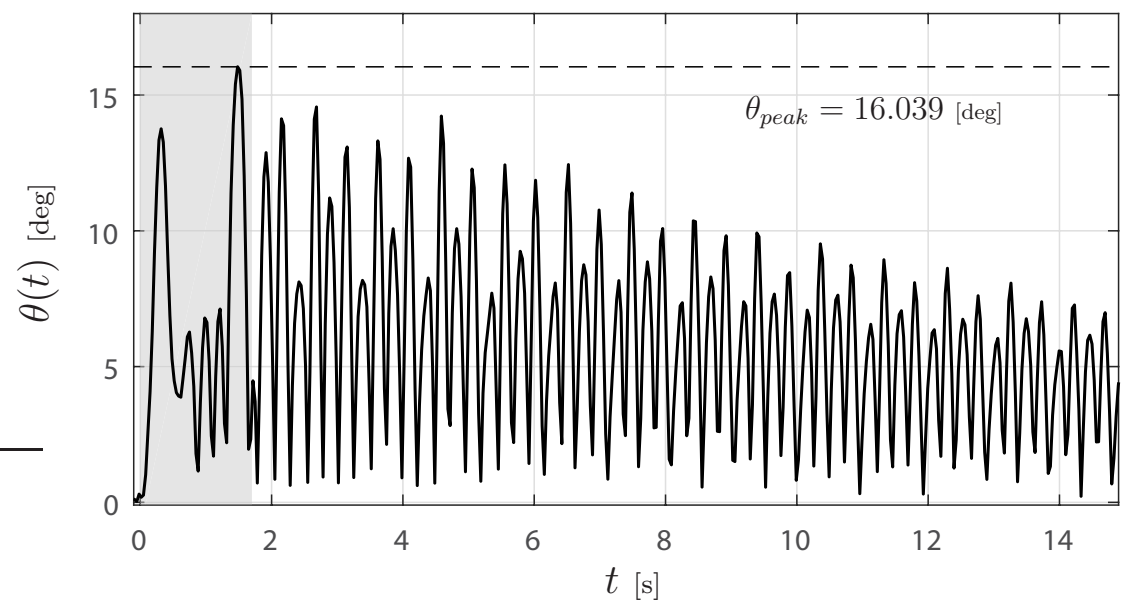

Figure 12: Polar angle $\theta$ when the vessel, loaded with 3 litres of water, is commanded with the non-filtered trajectory shown in Figure 11. The grey zone indicates the time duration of the motion.

\begin{tabular}{ccccccc}
\hline & & \multicolumn{2}{c}{$\omega_{n 1}[\mathrm{rad} / \mathrm{s}]$} & & \multicolumn{2}{c}{$\delta_{1}$} \\
\cline { 6 - 7 } Vol. [1] & $h[\mathrm{~m}]$ & Model & Exp. & & Model & Exp. \\
\hline 1 & 0.032 & 10.180 & 10.348 & & 0.0052 & 0.0087 \\
2 & 0.067 & 12.563 & 12.814 & & 0.0037 & 0.0061 \\
3 & 0.100 & 13.306 & 13.076 & & 0.0033 & 0.0050 \\
\hline
\end{tabular}

Table 1: Comparison between the parameters experimentally obtained for different liquid volumes and their theoretical values derived from equations (11) and (2). In the experiments, a cylindrical container of radius $R=97.5 \mathrm{~mm}$ filled with 1,2 and 3 litres of water with kinematic viscosity $\nu=1,004^{-6} \mathrm{~m}^{2} / \mathrm{s}$ is utilized.

confidence to tune the parameters of the trajectory filter.

\subsection{Testing the system on a point-to-point motion task}

The set of experimental tests described in this subsection looks at a simple point-to-point motion. Specifically, Figure 11 depicts the trajectories used in the tests: firstly, the system is fed with a non-filtered double-S velocity trajectory; secondly, the same double-S trajectory is filtered using the expo- 
Without tilting compensation

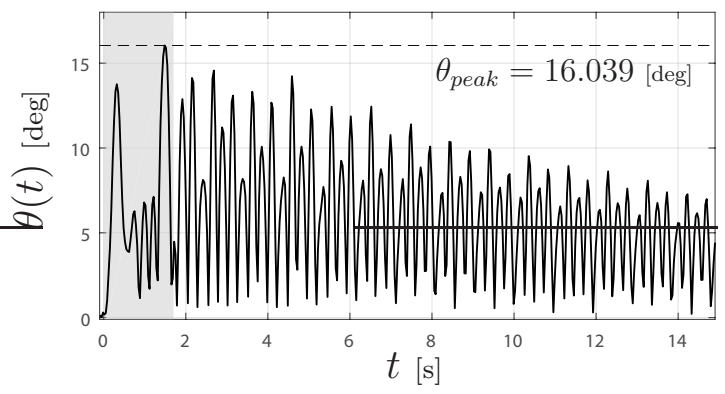

(a.1)
With tilting compensation

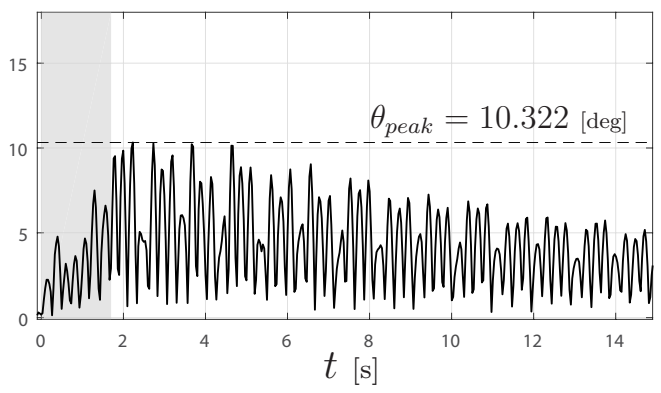

(a.2)

(a) Non-filtered trajectory: $\mathbf{p}^{\star}(t)$

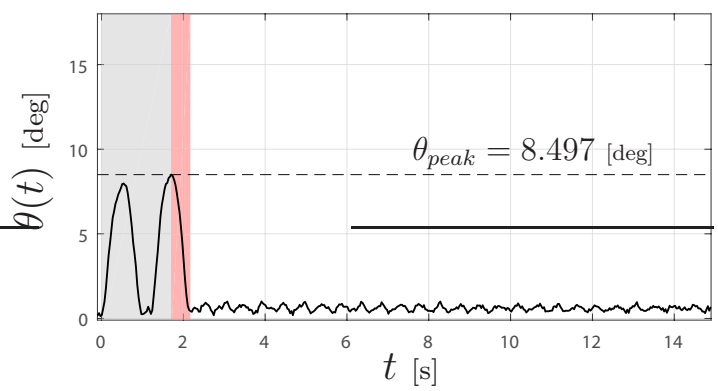

(b.1)

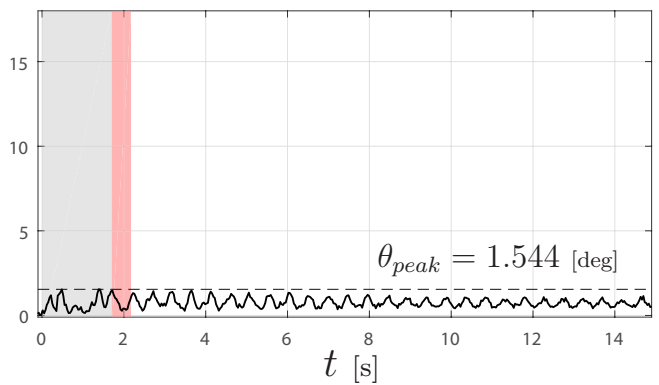

(b.2)

(b) Trajectoy filtered by the exponential filter: $\hat{\mathbf{p}}_{\text {exp }}^{\star}(t)$

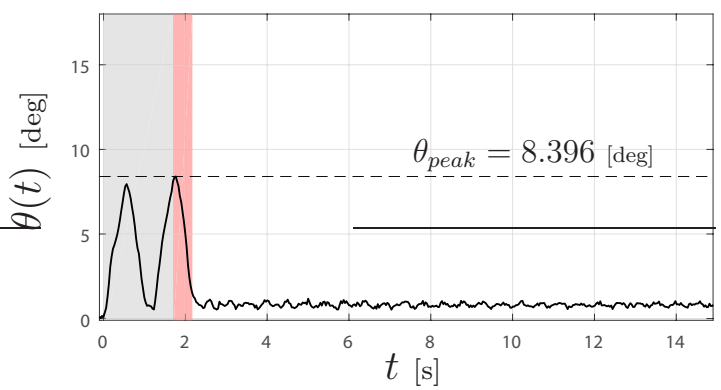

(c.1)

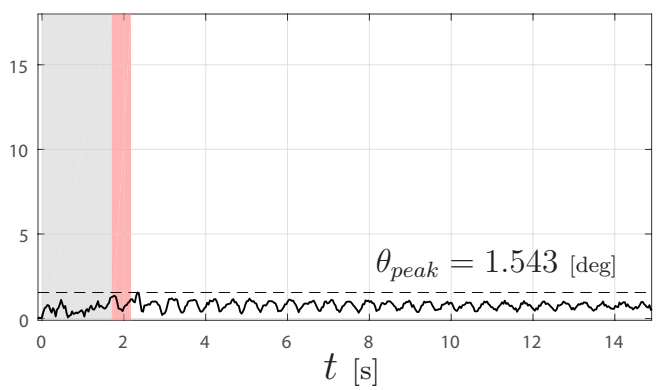

(c.2)

(c) Trajectoy filtered by the ZVD input shaper: $\hat{\mathbf{p}}_{z v d}^{\star}(t)$

Figure 13: Derived sloshing angle $\theta$ when a simple point-to-point motion is applied. The three inputs shown in Figure 11 are here used: (a) the non-filtered double-S velocity trajectory; (b) the trajectory filtered using the exponential smoother $F_{\text {exp }}(z)$; (c) the trajectory filtered by the ZVD Input Shaper $F_{z v d}(z)$. In the left column the tilting compensation mechanism is disabled, while in the right column this functionality is enabled. The red and grey zones indicate the time duration of the motion, respectively with and without filtering. 
nential smoother $F_{\exp }(z)$; finally, the initial double-S is filtered by the ZVD Input Shaper $F_{z v d}(z) 4$.

Considering the case of the robot loaded with 3 litres of water, Figure 13 shows the comparison between the three trajectories (figures11(a), 11(b) and 11(c) respectively) both when tilting compensation is not used (left column) and when this functionality is enabled (right column).

Looking at the left column in Figure 13, the reader can appreciate that both the exponential filter and the ZVD input smoother achieve similar results in terms of sloshing reduction, as they both guarantee approximately $50 \%$ reduction of the peak angle $\theta$. In addition, it is important to emphasize that the filtering actions are highly beneficial to reduce sloshing in steady state, while they are not effective when the robot moves. As a matter of fact, as discussed in Section 3, the large oscillations of angle $\theta$ during the robot motion cannot be cancelled, as they depend on the liquid inertia and the instantaneous acceleration imposed to the vessel by the robot.

Moving on to the right column in Figure 13, the reader can observe that a complete sloshing suppression can only be achieved by enabling the tilting compensation mechanism described in Section 3.2. This additional control action is able to nullify the effect of the robot acceleration on water. Importantly, the trajectory smoothing action is still necessary because the tilting compensation algorithm does not provide a robust solution, as it largely depends on the system parameters. Furthermore, the use of the sole tilting compensation mechanism (without any filtering action) would require a

\footnotetext{
${ }^{4}$ Filters $F_{\text {exp }}(z)$ and $F_{z v d}(z)$ are tuned according to the model described in Section 2 using the parameters listed in Table 1).
} 
higher order reference function to obtain a sufficiently smooth orientation trajectory, i.e. a feasible trajectory for the robot to track. Finally, Figure 13)(a.2) shows that the tilting compensation mechanism is able to compensate for the oscillations only during the robot motion, while a poorly damped oscillation in steady state at the frequency of the first sloshing mode can be still observed.

Overall, the performances of the combined filtering and tilting compensation actions can be evaluated from figures 13)(b.2) and 13)(c.2). In both cases, the sloshing effect is reduced by more than $90 \%$ with respect to the non-compensated case described in Figure 13(a.1). Although the two compared filters show similar performances, it is important to note that, while the ZVD input shaper shows a high degree of robustness with respect to the plant natural frequency and damping ratio (as proved in Biagiotti et al. $(2015)$ ), the exponential filter provides a smoother trajectory that can be better tracked by the robot. The latter property is fundamentally important, as the tilting compensation action is derived by computing the vessel orientation on the basis of the motion accelerations, thus benefiting from a higher continuity level of the filtered trajectory. This result is supported by Figure 14 which depicts the vessel orientation reference $\theta^{\star}$ computed according to equation (13) in the following situations: non-filtered trajectory (dashed line); trajectory filtered using the exponential smoother (solid line); and trajectory filtered by the ZVD Input Shaper (dashdot line). Figure 14 shows that both the non-filtered trajectory and the ZVD shaped trajectory present a discontinuous velocity profile, resulting in non-negligible tracking errors. Conversely, the trajectory smoothed by the exponential filter is char- 

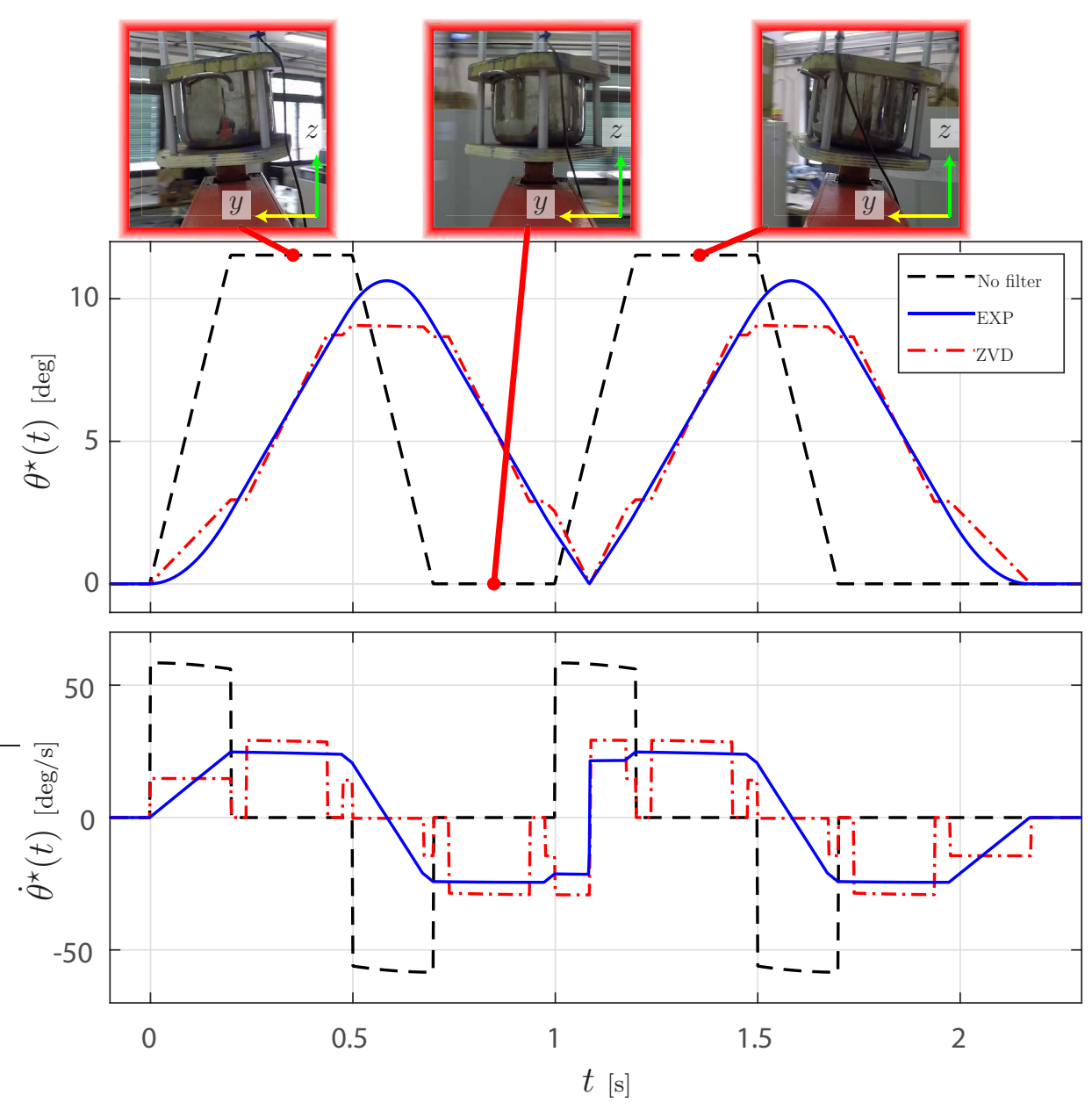

Figure 14: Vessel orientation reference $\theta^{\star}$ computed according to equation (13) in the following situations: non-filtered double-S velocity trajectory (dashed line); trajectory filtered using the exponential smoother (solid line); and trajectory filtered by the ZVD Input Shaper (dashdot line).

acterized by a continuous velocity profile. It is worth highlighting that the velocity discontinuity that seems to affect the trajectory generated by the exponential filter is due to the spherical coordinates representation when the sign of the azimuth angle $\varphi^{\star}$ changes.

The good performances of the exponential filter are also proved by Figure 


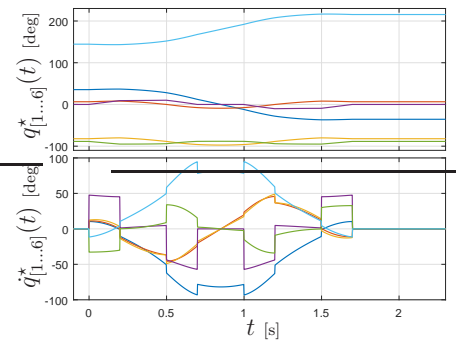

(a)

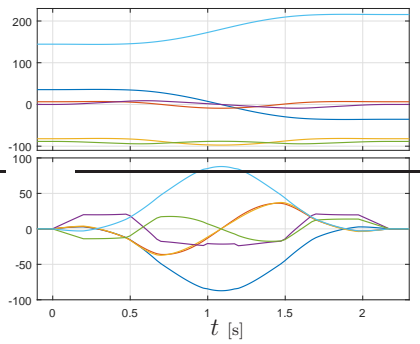

(b)

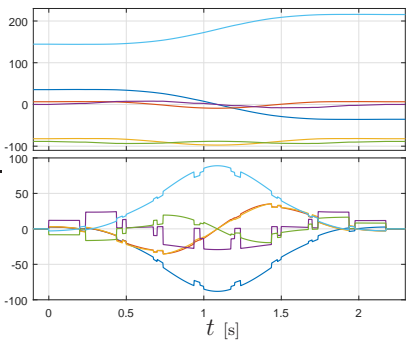

(c)

Figure 15: Position and velocity profiles commanded to the robot joints $q_{[1 \ldots 6]}$ in the scenario described in figures 13 and 14 non-filtered double-S velocity trajectory (a); trajectory filtered using the exponential smoother (b); and trajectory filtered by the ZVD Input Shaper (c).

15 that shows the six joint trajectory profiles derived from the inverse kinematics function. The reader can observe that, because of the discontinuities on the desired angular velocity of the end-effector, the non-filtered trajectory and the ZVD filtered trajectory lead to discontinuous joint velocities. On the contrary, the exponential filter produces continuous joint velocity profiles.

Furthermore, the exponential filter and its low-pass characteristics allow for better performances, as not only does the filter suppress the vibration at the required specific frequency, but it also reduces potential resonant modes located at higher frequencies. This property is illustrated by Figure 16, where the sensitivity curv 5 of the exponential smoother and the ZVD input shaper are compared. In particular, Figure [16 shows the sensitivity of the filters with respect to the natural frequency of the system when the damping ratio $\delta$ is equal to its nominal value $\delta_{1}=0.0050$. The reader can observe that,

\footnotetext{
${ }^{5}$ By definition, the sensitivity curve represents the percentage of the residual vibration induced on the vibrating plant by the shaped input with respect to the vibration caused by the unfiltered signal as a function of the system parameters, see Singh and Singhose (2002).
} 


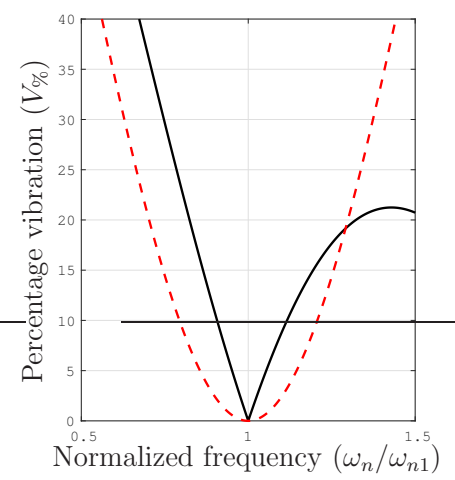

(a)

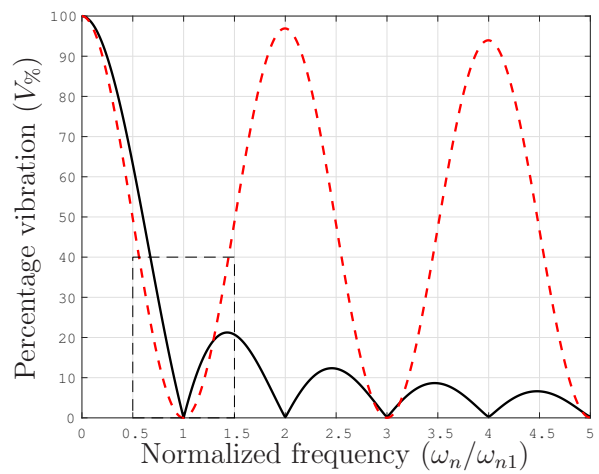

(b)

Figure 16: Sensitivity curve of the exponential smoother (black solid line) and the ZVD input shaper (red dashed line) with respect to the natural frequency $\omega_{n}$ : residual vibration around the nominal value $\omega_{n 1}$ (a) and residual vibration for large variations of $\omega_{n}$ (b).

while the sensitivity curve of the exponential smoother lies over the curve of the ZVD input shaper for small variations of $\omega_{n}$ around its nominal value $\omega_{n 1}$, the two sensitivity curves largely differ for large variations of $\omega_{n}$ (see Figure 16(a)d. Specifically, the sensitivity curve of the exponential smoother rapidly decreases as $\omega_{n}$ increases, allowing the compensation of non-modelled higher order modes. Therefore, while the exponential smoother guarantees an effective cancellation of the vibrations caused by these additional modes, the ZVD input shaper does not guarantee this property.

To conclude, it is important to note that the measured angle $\theta$ in Figure 13 never becomes zero, despite its oscillatory model. This may be due to a number of different factors, most importantly: (i) the camera readings used to measure the liquid surface configuration is noisy; (ii) the used theoretical model is based on the assumption that the liquid surface is a plane; (iii) the sloshing model presented in Section 2 assumes that the centre of the liquid 
surface remains fixed during the motion, while model (16) - used to derive the configuration of the liquid surface from the measures of the camera introduces a translational term $\Delta h$ that is different from zero.

\subsection{Testing the system on a complex multi-point motion task}

This subsection presents a set of tests aimed at evaluating the proposed solution in a more realistic scenario. Specifically, a typical industrial task is simulated, where the vessel must be moved through a set of workstations. To this aim, the robot is commanded with a cubic B-Spline trajectory that models a complex motion composed of a set of $3 \mathrm{D}$ segments and stop points. In this regard, it is important to recall that B-Splines are de-facto the standard tools used in industrial applications to plan complex motions interpolating a set of via-points $\mathbf{p}_{i}^{\star}, i=0, \ldots, n-1$ and related time instants $t_{i}$.

This work deals with uniform B-splines trajectories, i.e. B-splines characterized by an equally-spaced distribution of the knots $t_{i}$ such that $t_{i+1}-t_{i}=$ $T, \quad i=0, \ldots n-2$ (see Biagiotti and Melchiorri (2008)). Consequently, as proved in Biagiotti and Melchiorri (2010) and Biagiotti and Melchiorri (2013), the 3D trajectory can be generated on-the-fly by means of a chain of discrete FIR filters. It is necessary to emphasize that the B-spline generator provides the position, velocity and acceleration profiles required by the tilting compensation algorithm.

Figure 17 shows the geometrical path of the B-Spline trajectory used during the tests. The grey circles denote the positions where the motion stops for a few seconds. Similarly to the case of the point-to-point trajectory, the following situations have been tested: the non-filtered B-spline trajectory (black line); the B-spline trajectory filtered using the exponential smoother 


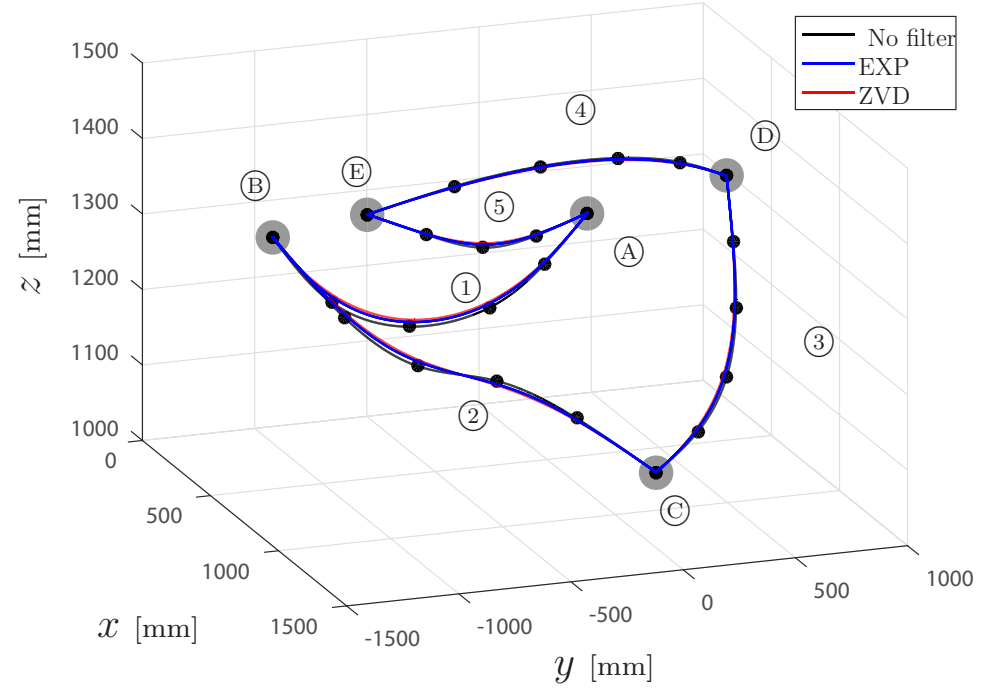

Figure 17: Geometrical path of the planned B-spline trajectory used in the multi-point tests: non-filtered B-spline trajectory (black line); B-spline trajectory filtered using the exponential smoother (blue line); B-spline trajectory filtered by the ZVD input shaper (red line). Motion segments are labelled with numbers, whereas stop-points are labelled with letters.

(blue line); and the B-spline trajectory filtered by the ZVD input shaper (red line). The reader can observe that the cubic B-spline trajectory has the same continuity level as the double-S trajectory used for the point-to-point tests; therefore, any consideration about the continuity of the orientation trajectory is still valid.

Figure 18 shows the speed profiles along the motion segments in case of the non-filtered trajectory (dashed line), the trajectory filtered through the exponential smoother (solid line), and the trajectory filtered by the ZVD Input Shaper (dashdot line). Figure 19 depicts the sloshing angles when the filtered and non-filtered B-spline trajectories are applied to the system. The geometric deformation and the $T$ delay introduced by the two filters are noticeable from figures 18 and 19. However, despite the complex 3D motion 


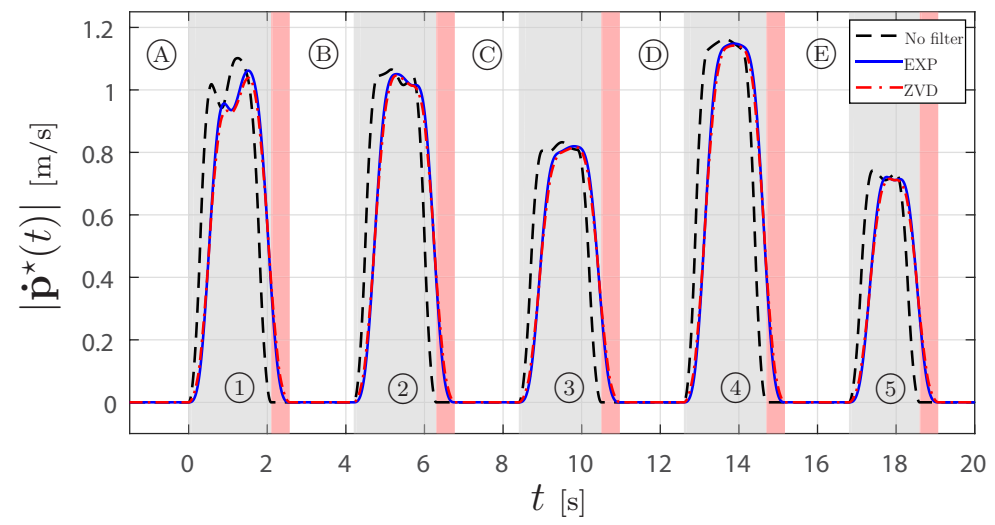

Figure 18: Velocity profiles corresponding to the trajectories shown in Figure17. The grey zones indicate the B-spline trajectory motion segments, whereas the delay introduced by the filters is highlighted in red.

and the very high speeds imposed to the vessel, the proposed feed-forward controller is able to significantly reduce the sloshing phenomenon both along motion segments and when the robot stops.

Figure 19] shows that the exponential smoother allows approximately $80 \%$ reduction of sloshing, whereas the ZVD input shaper reduces sloshing by approximately $86 \%$. As discussed in Section 4.3, the greater robustness of the input shaper with respect to parameter uncertainties accounts for the fact that the ZVD input shaper slightly outperforms the exponential smoother in terms of sloshing compensation. However, the exponential smoother allows a better compensation of non-modelled higher order modes because of its smaller sensitivity function for large $\omega_{n}$ values, which makes it preferable in industrial applications.

The available featured video shows the performances of the proposed feedforward controller in the scenario described above. 


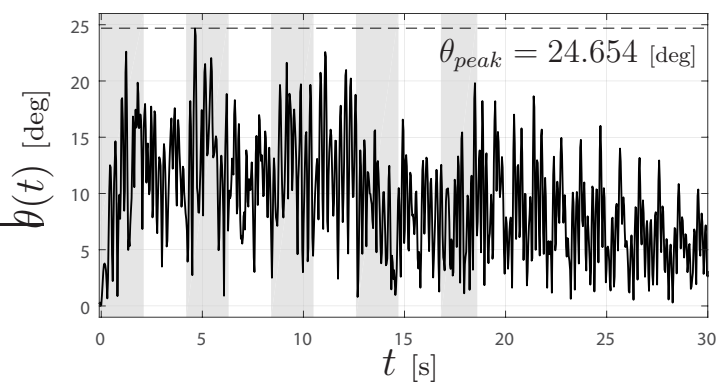

(a)

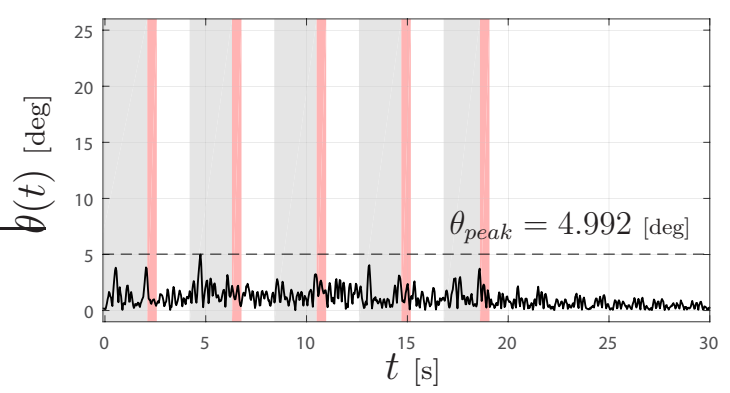

(b)

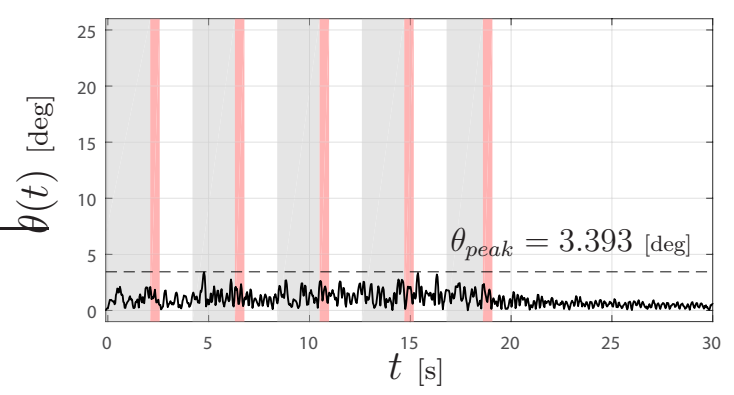

(c)

Figure 19: Sloshing angle profiles corresponding to a vessel filled with 3 litres of water and moved according to the trajectories depicted in Figure 17; non-filtered B-spline trajectory (a); B-spline trajectory filtered using the exponential smoother (b); B-spline trajectory filtered by the ZVD input shaper (c).

\section{Conclusions}

This paper addresses the problem of using a robot manipulator to move a vessel filled with liquid. It presents a feed-forward method able to consid- 
erably reduce sloshing phenomena by exploiting all the degrees of freedom of the robot manipulator.

Specifically, a two-stage controller is proposed, which combines a filtering action on the original desired motion trajectory with a tilting compensation algorithm that counteracts the robot lateral accelerations. To compensate sloshing, first its dynamics are modelled by means of a spherical pendulum equivalent model and then a vibration suppression exponential filter is used to counteract the liquid waves. Finally, a feed-forward controller is designed to compensate the robot accelerations and regulate the vessel orientation.

The exhaustive range of experimental tests presented in the paper demonstrates the effectiveness of the proposed controller, which reduces the sloshing dynamics by more than $70 \%$ in different operating conditions. The experimental results show that while the exponential filter only acts at the end of the motion - i.e., on the residual oscillation caused by the robot stopping - the tilting compensation controller is able to compensate the sloshing dynamics during the robot motion. In light of this result, the two controllers are complementary and must be combined in the same architecture. Furthermore, the exponential filter is beneficial to the tilting compensation controller because it increases the trajectory smoothness.

To conclude, it is important to emphasize that the proposed method can be effectively implemented in many industrial applications, as the complexity of the solution is limited both from a computational and a technological point of view. As a matter of fact, the feed-forward controller can be easily implemented in standard industrial robotics systems without the need for any additional equipment or modification of the robot control system. 


\section{Acknowledgements}

This work was supported by the UK Engineering and Physical Sciences Research Council (EPSRC) [grant number EP/R005524/1] and by Innovate UK [grant number 102908].

Abramson, H., 1966. The Dynamic Behavior of Liquids in Moving Containers: With Applications to Space Vehicle Technology. NASA SP. Scientific and Technical Information Division, NASA.

Aribowo, W., Yamashita, T., Terashima, K., Jan 2015. Integrated trajectory planning and sloshing suppression for three-dimensional motion of liquid container transfer robot arm. Journal of Robotics, $1-15$.

Aribowo, W., Yamashita, T., Terashima, K., Kitagawa, H., Oct 2010. Input shaping control to suppress sloshing on liquid container transfer using multi-joint robot arm. In: Intelligent Robots and Systems (IROS), 2010 IEEE/RSJ International Conference on. pp. 3489-3494.

Besset, P., Béarée, R., 2017. Fir filter-based online jerk-constrained trajectory generation. Control Engineering Practice 66, 169 - 180.

Biagiotti, L., Melchiorri, C., 2008. Trajectory Planning for Automatic Machines and Robots. Springer Berlin Heidelberg.

Biagiotti, L., Melchiorri, C., May 2010. B-spline based filters for multi-point trajectories planning. In: Robotics and Automation (ICRA), 2010 IEEE International Conference on. Anchorage, Alaska, pp. 3065-3070. 
Biagiotti, L., Melchiorri, C., 2012. FIR filters for online trajectory planning with time- and frequency-domain specifications. Control Engineering Practice 20 (12), $1385-1399$.

Biagiotti, L., Melchiorri, C., Nov 2013. Online trajectory planning and filtering for robotic applications via b-spline smoothing filters. In: IEEE/RSJ International Conference on Intelligent Robots and Systems (IROS). pp. $5668-5673$.

Biagiotti, L., Melchiorri, C., Moriello, L., 2015. Optimal trajectories for vibration reduction based on exponential filters. Control Systems Technology, IEEE Transactions on 24 (2), $609-622$.

Chen, S. J., Hein, B., Worn, H., April 2007. Using acceleration compensation to reduce liquid surface oscillation during a high speed transfer. In: Proceedings 2007 IEEE International Conference on Robotics and Automation. pp. 2951-2956.

Consolini, L., Costalunga, A., Piazzi, A., Vezzosi, M., Nov 2013. Minimumtime feedforward control of an open liquid container. In: IECON 2013 39th Annual Conf. of the IEEE. pp. 3592-3597.

Feddema, J. T., Dohrmann, C. R., Parker, G. G., Robinett, R. D., Romero, V. J., Schmitt, D. J., Feb 1997. Control for slosh-free motion of an open container. IEEE Control Systems 17 (1), 29-36.

Hamaguchi, M., Taniguchi, T., 2002. Transfer control and curved path design for cylindrical liquid container. In: 15th IFAC World Congress. Vol. 35. pp. $79-84$. 
Hamaguchi, M., Taniguchi, T., 2009. Sloshing damping control in a cylindrical container on a wheeled mobile robot using dual-swing active-vibration reduction. J. of Robotics and Mechatronics 21 (5), 642-646.

Ibrahim, R., 2005. Liquid Sloshing Dynamics: Theory and Applications. Cambridge University Press.

Moriello, L., Biagiotti, L., Melchiorri, C., Paoli, A., May 2017. Control of liquid handling robotic systems: A feed-forward approach to suppress sloshing. In: Robotics and Automation (ICRA), 2017 IEEE International Conference on. Singapore.

Nair, I., February 2017. Robotic chef creates recipe for change. URL http://fstjournal.org/features/31-1/robotic-chef

Singer, N., Singhose, W., Seering, W., 1999. Comparison of filtering methods for reducing residual vibration. European Journal of Control 5 (2), 208 218.

Singh, T., Singhose, W., 2002. Tutorial on input shaping/time delay control of maneuvering flexible structures. In: In American Control Conference.

Yano, K., Terashima, K., May 2001. Robust liquid container transfer control for complete sloshing suppression. IEEE Transactions on Control Systems Technology 9 (3), 483-493.

Yano, K., Terashima, K., Feb 2005. Sloshing suppression control of liquid transfer systems considering a 3-d transfer path. IEEE/ASME Transactions on Mechatronics 10 (1), 8-16. 
Supplementary Material - video
Click here to download Supplementary Material for on-line publication only: complete_sloshing_tilt.mp4

Supplementary Material - video
Click here to download Supplementary Material for on-line publication only: complete_sloshing_tilt.mp4

\section{4}

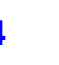

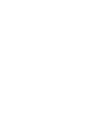

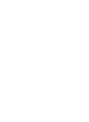

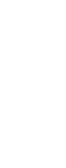
. .

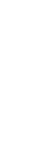

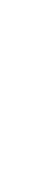

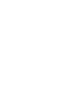

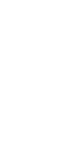

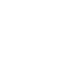

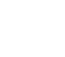

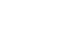

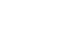

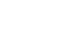

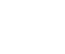

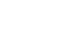

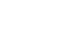

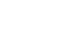

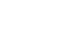

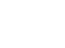

